\title{
Erlangen Score predicts cognitive and neuroimaging progression in mild cognitive impairment stage of Alzheimer's disease
}

Tobias Skillbäck ${ }^{1}$, Johannes Kornhuber ${ }^{2}$, Kaj Blennow ${ }^{1,3}$, Henrik Zetterberg ${ }^{1,3,4,5}$, Piotr Lewczuk $^{2,6}$, for the Alzheimer's Disease Neuroimaging Initiative*

Running title: Erlangen score predicts progression in MCI

* Data used in preparation of this article were obtained from the Alzheimer's Disease Neuroimaging Initiative (ADNI) database (adni.loni.usc.edu). As such, the investigators within the ADNI contributed to the design and implementation of ADNI and/or provided data but did not participate in analysis or writing of this report. A complete listing of ADNI investigators can be found at: http://adni.loni.usc.edu/wpcontent/uploads/how to_apply/ADNI_Acknowledgement_List.pdf (also available as supplemental material).

${ }^{1}$ Institute of Neuroscience and Physiology, Department of Psychiatry and Neurochemistry, the Sahlgrenska Academy at the University of Gothenburg, Mölndal, Sweden

${ }^{2}$ Department of Psychiatry and Psychotherapy, Universitätsklinikum Erlangen, and Friedrich-Alexander Universität Erlangen-Nürnberg, Erlangen, Germany

${ }^{3}$ Clinical Neurochemistry Laboratory, Sahlgrenska University Hospital, Mölndal, Sweden

${ }^{4}$ Department of Molecular Neuroscience, UCL Institute of Neurology, London WC1N 3BG, $U K$

${ }^{5} U K$ Dementia Research Institute at UCL, London WC1N 3BG, UK

${ }^{6}$ Department of Neurodegeneration Diagnostics, Medical University of Bialystok, Poland 
Corresponding author: Tobias Skillbäck, Clinical Neurochemistry Laboratory, Sahlgrenska University Hospital/Mölndal, S-431 80 Mölndal, Sweden. Telephone: +46 31 3430025. E-mail: tobias.skillback@gu.se

Date of revision: 22/01-2019

Keywords: Alzheimer disease, Erlangen score, Cerebrospinal Fluid, biomarkers, Abeta(1-42), T-tau, P-tau. 


\begin{abstract}
Background: To alleviate the interpretation of the core Alzheimer's disease (AD) cerebrospinal fluid (CSF) biomarkers amyloid $\beta_{1-42}(\mathrm{~A} \beta 42)$, total tau (T-tau) and phosphorylated tau (P-tau), the Erlangen Score (ES) interpretation algorithm has been proposed.
\end{abstract}

Objective: In this study, we aim to assess the predictive properties of the ES algorithm on cognitive and neuroimaging outcomes in mild cognitive impairment (MCI).

Methods: All MCI subjects with an available baseline CSF sample from ADNI-1 were included ( $n=193$ ), and assigned an ES between 0 and 4 based on their baseline CSF biomarker profile. Structural magnetic resonance imaging brain scans and MMSE and ADAS-Cog scores were collected at up to 7 times in follow-up examinations.

Results: We observed strong and significant correlations between the ES at baseline and neuroimaging and cognitive results with patients with neurochemically probable AD (ES =4) progressing significantly $(\mathrm{p} \leq 0.01)$ faster than those with a neurochemically improbable AD $(\mathrm{ES}=0$ or 1$)$, and the subjects with neurochemically possible $\mathrm{AD}(\mathrm{ES}=2$ or 3$)$ in-between these two groups.

Conclusion: This study further demonstrates the utility of the ES algorithm as a as a tool in predicting cognitive and imaging progression in MCI patients. 


\section{Introduction}

The importance of cerebrospinal fluid (CSF) biomarkers of Alzheimer's disease (AD) as an aid for diagnostic decisions and a measure of disease progression is growing, both in research and in the clinical setting [1-3]. Reaching early and dependable diagnosis and prognosis for patients with memory complaints and other cognitive symptoms is likely key to be able to in time administer future treatments minimizing irreparable damage to the central nervous system [4]. A well-established panel of CSF biomarkers might be able to provide needed support in this task. The four most prominent and widely used biomarkers to date reflect different aspects of AD pathology: deposition of amyloid plaques leading to decreased concentrations of CSF amyloid $\beta_{1-42}(\mathrm{~A} \beta 42)$ and decreased $\mathrm{A} \beta 42 / 40$ ratio; neuronal degeneration as reflected by total tau (T-tau); and neurofibrillary tangle formation correlated to phosphorylated tau (P-tau) [5, 6]. However, inter-laboratory differences in biomarkers assays, analytical procedures and pre-analytical sample collection protocols have made it hard to establish laboratory-independent cutoff values [7]. This leads to difficulties in comparing CSF results between centers, and even within a given center if a measurement method and/or preanalytical handling protocol are changed. To alleviate the interpretation of laboratory analysis results in $\mathrm{AD}$, the Erlangen Score (ES) interpretation algorithm (ES) has previously been proposed [8]. The ES categorizes subjects into five ordinal categories with scores from 0 to 4 based on their CSF biomarker profile in relation to cutoff values that are given laboratory-specific. This approach reflects reality closer than the dichotomization into normal or pathologic biomarker status used in other studies $[9,10]$. The ES algorithm has previously been validated in pre-dementia subjects in two multicenter studies, and its utility was supported by strong associations to the development of AD dementia (ADD) [1113]. However, the relation of the ES to cognitive and neuroradiological measures of the disease progression in mild cognitive impairment (MCI) stage of AD has never been investigated. 
Therefore, in this study we aimed to assess the validity of the ES algorithm as a tool for predicting cognitive and imaging progression in MCI. We hypothesized that a higher score would be associated with faster disease progression.

\section{Methods}

Data used in the preparation of this article were obtained from the Alzheimer's disease Neuroimaging Initiative (ADNI) database (adni.loni.usc.edu). ADNI was launched in 2003 by the National Institute on Aging (NIA), the National Institute of Biomedical Imaging and Bioengineering (NIBIB), the Food and Drug Administration (FDA), private pharmaceutical companies and non-profit organizations as a $\$ 60$ million, 5-year public-private partnership. The primary goal of ADNI has been to test whether serial magnetic resonance imaging (MRI), positron emission tomography (PET), other biological markers, and clinical and neuropsychological assessment can be combined to measure the progression of MCI and early AD. The Principal Investigator of this initiative is Michael W. Weiner, MD, VA Medical Center and University of California - San Francisco. ADNI is the result of efforts of many coinvestigators from a broad range of academic institutions and private corporations, and subjects have been recruited from over 50 sites across the U.S. and Canada. The initial goal of ADNI was to recruit 800 subjects but ADNI has been followed by ADNI-GO and ADNI-2. To date these three protocols have recruited over 1500 adults, ages 55 to 90 , to participate in the research, consisting of cognitively normal older individuals, people with early or late MCI, and people with early AD. The follow up duration of each group is specified in the protocols for ADNI-1, ADNI-2 and ADNI-GO. Subjects originally recruited for ADNI-1 and ADNI-GO had the option to be followed in ADNI-2. For up-to-date information, see www.adni-info.org, where inclusion/exclusion criteria are also described in detail. 


\section{Subjects}

Our study population consisted of all MCI subjects with available baseline CSF samples from ADNI-1 ( $n=193)$. Briefly, all subjects included in ADNI-1 were between the ages of 55 and 90 years, had completed at least 6 years of education, were fluent in Spanish or English, and were free of any significant neurologic disease other than AD. The MCI group had Mini Mental State Examination (MMSE) score $\geq 24$, objective memory loss as shown on scores on delayed recall of the Wechsler Memory Scale Logical Memory II (>1 standard deviations below the normal mean), Clinical Dementia Rating scale 0.5, preserved activities of daily living, and absence of dementia.

\section{Erlangen Score}

All study subjects were assigned an ES between 0 and 4 based on their CSF biomarker profile with a macro written in Microsoft Excel. The detailed protocol for the ES can be found elsewhere [8]. Briefly, a CSF profile with all biomarkers normal is scored 0 points; a pattern with only slight alterations in one biomarker group (either $A \beta$ or Tau, but not both) results in the score of 1 ; alterations in amyloid $\beta$ pathology (in this particular study, decreased $A \beta 1-42$ concentrations) or tau metabolism (increased concentrations of T-tau and/or P-tau) but not in both pathologies is scored 2 points; a result with clear alterations in one biomarkers' group (either $\mathrm{A} \beta$ or Tau) accompanied by marginal alterations in the other group is scored 3 points; clear alterations in both $\mathrm{A} \beta$ and T-tau/P-tau result in 4 points. A simplified rendition of the interpretation algorithm used to translate the biomarker profiles of each subject is described elsewhere. Eventually, the subjects with the score of 0-1 are defined as "neurochemically improbable AD", the subjects with the score of 2-3 are defined as "neurochemically possible AD", and the subjects with the score of 4 are defined as "neurochemically probable AD". 


\section{CSF measurements}

CSF collection, processing and storage procedures have been described previously [14]. CSF A $\beta 42$, T-tau and P-tau were measured using the multiplex xMAP Luminex platform (Luminex Corp, Austin, TX, USA) with the INNOBIA AlzBio3 kit (Fujirebio, Ghent, Belgium). The reference ranges used for the calculations of the scores were taken directly from the previous study [14], and applied for this study without further modifications: 192 $\mathrm{pg} / \mathrm{mL}$ for $\mathrm{A} \beta 1-42,93 \mathrm{pg} / \mathrm{mL}$ for T-tau, and $23 \mathrm{pg} / \mathrm{mL}$ for P-tau. The border zones, defining "slight alterations", were set as the concentrations within the range of $173-192 \mathrm{pg} / \mathrm{mL}$ for A $\beta 1-42$, within 93-102 pg/mL for T-tau, and within $23-25 \mathrm{pg} / \mathrm{mL}$ for P-tau.

\section{Magnetic resonance imaging}

Structural magnetic resonance imaging brain scans were acquired using 1.5 Tesla MRI scanners (up to 7 time points: screening, and 6, 12, 18, 24, 36 and 48 months) with a standardized protocol including T1-weighted MRI scans using a sagittal volumetric magnetization prepared rapid gradient echo (MP-RAGE) sequence [15]. In brief, automated volume measures were performed with FreeSurfer software package (http://surfer.nmr.mgh.harvard.edu/fswiki) $[16,17]$. We used averaged volume measurements for the right and left hippocampi for this study.

\section{Cognitive assessments}

Overall cognition was assessed by the Mini Mental State Examination (MMSE) and the Alzheimer's Disease Assessment Scale-Cognitive Subscale (ADAS-Cog) tests at up to 7 time points (screening, and at 6,12, 18, 24, 36 and 48 months after the baseline assessment). 


\section{Statistical analysis}

If not stated otherwise, the results of continuous variables are presented as means and standard deviations (std. dev.) or as medians and inter-quartile ranges (IQR). ANOVA and $\chi 2$ statistics were used to test differences in age and sex distribution, baseline measurements of hippocampal and whole brain volumes, and baseline MMSE and ADAS-Cog scores between the study groups. Generalized Estimating Equation (GEE) was used to discern statistical differences between ES groups in the development of MMSE scores, ADAS-Cog scores, hippocampal volumes and whole brain volumes over time. The interaction term of ES*month from baseline was used to test modifying effects of the ES on the effect of the observation time. All longitudinal changes in MMSE, ADAS-Cog scores, whole brain volumes, and hippocampal volumes were normalized to a percentage change in relation to their baseline values. Statistical significance was determined at the $\mathrm{p}<0.05$ level. All statistics, charts, and tables were generated with SPSS version 22 (IBM, New York, USA).

\section{Ethics}

All CSF samples analyzed in this study were collected in accordance with regional ethical guidelines as well as the ethical standards of the Helsinki Declaration of 1975.

\section{Results}

Demographics of the study cohort

Demographics and the baseline measures of the metrics analyzed in this study are presented in table 1 . There were no significant differences in age between the groups. There were significant differences in the sex distribution between the groups $(\mathrm{p}=0.02)$. The group with neurochemically improbable $\mathrm{AD}(\mathrm{ES}=0$ or 1$)$ had $26 \%$ female subjects, the group with 
neurochemically possible $\mathrm{AD}(\mathrm{ES}=2$ or 3$)$ had $18 \%$ female subjects, and the group with neurochemically probable $\mathrm{AD}(\mathrm{ES}=4)$ had $40 \%$ female subjects.

Subjects in the neurochemically improbable AD group had significantly larger hippocampal volumes at baseline compared to the neurochemically possible (mean diff $=718 \mathrm{~mm}^{3}, \mathrm{p}=$ 0.03 ) and the neurochemically probable (mean diff $=777 \mathrm{~mm}^{3}, \mathrm{p}=<0.01$ ) AD groups. There were no significant difference in hippocampal volumes between the neurochemically possible and probable groups $(\mathrm{p}=1.0)$.

The neurochemically improbable AD subjects also had significantly larger whole brain volumes at baseline compared to those with neurochemically probable $\mathrm{AD}$ (mean diff $=6.9 \mathrm{E} 5$ $\left.\mathrm{mm}^{3}, \mathrm{p}<0.01\right)$ but not compared to the subjects with neurochemically possible AD $(\mathrm{p}=1.0)$. The neurochemically possible group and the neurochemically probable group did not significantly differen in baseline whole brain volumes $(\mathrm{p}=0.11)$.

The neurochemically improbable AD group registered lower ADAS-Cog scores at baseline compared to the neurochemically possible $\mathrm{AD}$ (mean diff $=5.5, \mathrm{p}<0.01$ ) and the neurochemically probable $\mathrm{AD}$ (mean diff $=5.5, \mathrm{p}<0.01$ ) groups. No significant differences in ADAS-Cog scores between the neurochemically possible and probable AD groups could be found $(\mathrm{p}=1.0)$.

The neurochemically improbable AD group presented higher MMSE scores than the neurochemically possible AD (mean diff $=1.0, \mathrm{p}=0.04$ ) but not the neurochemically probable group $(\mathrm{p}=0.20)$. There were, again, no significant differences between the neurochemically possible and probable AD groups $(\mathrm{p}=0.64)$.

\section{Longitudinal changes in cognitive measures in the MCI group}

Follow-times and and number of participants at each point of follow-up is presented in table 2. MMSE scores declined significantly faster in the subjects with neurochemically possible 
$\mathrm{AD}(\mathrm{B}=-0.20, \mathrm{p}=0.03)$ and with neurochemically probable $\mathrm{AD}(\mathrm{B}=-0.36, \mathrm{p}<0.01)$ compared to the subjects with neurochemically improbable AD (fig. 1a). There was a nonsignificant tendency towards faster decline in the MMSE score in neurochemically probable subjects compared to neurochemically possible subjects $(\mathrm{B}=-0.16, \mathrm{p}=.10)$.

Subjects with neurochemically probable AD also increased faster in ADAS-Cog scores as compared to the subjects with improbable $\mathrm{AD}(\mathrm{B}=0.71, \mathrm{p}=0.01$, fig. $1 \mathrm{~b})$. We did not observe differences in ADAS-Cog scores increase between the neurochemically possible AD subjects compared to the neurochemically improbable AD subjects $(B=0.10, p=0.76)$. There were however a significant difference in decline between the neurochemically probably and neurochemically possible $\mathrm{AD}$ subjects $(\mathrm{B}=0.60, \mathrm{p}=0.02)$.

\section{Longitudinal changes in brain structure in MCI}

Whole brain volumes decreased faster in subjects with neurochemically possible AD $(B=-$ $0.032, \mathrm{p}=0.03)$ and neurochemically probable $\mathrm{AD}(\mathrm{B}=-0.066, \mathrm{p}<0.01)$ as compared to subjects with neurochemically improbable AD (fig. 1c). Whole brain volumes also decreased faster in subjects with neurochemically probable $\mathrm{AD}(\mathrm{B}=-0.034, \mathrm{p}=0.01)$ as compared to subjects with neurochemically possible AD.

Hippocampal volumes of patients with neurochemically possible $\mathrm{AD}(\mathrm{B}=-0.084, \mathrm{p}<0.01)$, and neurochemically probable $\mathrm{AD}(\mathrm{B}=-0.20, \mathrm{p}<0.01)$ decreased at a faster pace compared to the subjects with neurochemically improbable $\mathrm{AD}$ (fig. 1d). This was also true for subjects with neurochemically probable $\mathrm{AD}(\mathrm{B}=-0.117, \mathrm{p}<0.01)$ as compared to subjects with neurochemically possible AD. 


\section{Discussion}

In this study, we examined the utility of the Erlangen Score as a globally implementable composite $\mathrm{AD}$ biomarker evaluation algorithm to predict cognitive and brain imaging decline in MCI stage of $\mathrm{AD}$. We found that a higher ES predicted a faster disease progression in MCI patients; the subjects with higher ES showed a faster reduction of the whole brain and the hippocampal volumes, as well as faster decrease in MMSE, and a faster increase in ADASCog scores.

In all disease progress measures selected for this study there were clear and statistically significant distinctions between patients with neurochemically improbable AD (ES of 0-1) and those with neurochemically probable AD (ES of 4), and for most measures also between the subjects with neurochemically improbable AD and those with neurochemically possible $\mathrm{AD}(\mathrm{ES}=2$ or 3). Expectedly, subjects with ES 2-3 exhibited disease developing patterns in between those with the scores score $0-1$ and 4 . Here, the differences between the groups were statistically weaker, probably at least partly due to the low number of subjects with ES 2 and 3 ( $n=27$ and $n=7$, respectively). However, their statistical parameters in the GEE models as well as visual indications in the Loess regressions in figure 1 corroborate them being part of a continuum between the higher subject count groups of neurochemically improbable AD and neurochemically probable $(\mathrm{ES}=4) \mathrm{AD}$. An interesting feature of the differences in the correlations of the cognitive and the structural measures and ES, is that the neurochemically improbable $\mathrm{AD}$ and the neurochemically possible $\mathrm{AD}$ divert from each other in structural but not in cognitive measures. One explanation for this might be that, in $\mathrm{AD}$, structural decay can be radiologically visualized before cognitive decline can be detected by MMSE or ADASCog [18]. This means, that the subjects with no or only moderate CSF alterations (i.e. those 
with $\mathrm{ES}=0-3$ ) are most probably at a stage of the disease when the structural changes can already be seen, but the cognitive decline has not yet reached pathological levels. To our best knowledge, ES is the first modality/biomarker reported so far capable to predict as small as $10 \%$ drop in the MMSE score in a timespan of less than 24 months (see fig. 1a).

It should be noted that figure 1 is intended as an illustration, while the GEE model provide statistical proof of differences. Some seeming anomalies between the GEE results and the graphical representations of data in the local regression illustrations occur. For instance the local regression lines representing the MMSE progression of the improbable and the possible AD groups cross paths, but still differ significantly in the GEE model. This likely stem from the linear nature of the GEE model contrasting with the adaptive nature of the local regression, and also the low number of participants, particularly at follow up, in the neurochemically probable AD group.

Interestingly, in our recent study we found that MCI patients with neurochemically possible $\mathrm{AD}$ had, compared to the patients with neurochemically improbable AD, 6 - 8 times higher hazards to progress to the dementia stage of $\mathrm{AD}$ in the first three follow up years, and then their hazards decreased and became comparable to those with improbable $\mathrm{AD}$; on the other hand, MCI patients with neurochemically probable AD had hazards to develop AD dementia 8 - 12 times higher compared to the patients with neurochemically improbable AD [13].

The most serious limitation of this study is the unavailability of results of CSF A $\beta 1-40$ in this cohort, which precluded integration of the $A \beta 42 / 40$, a biomarker known to better reflect the amyloid $\beta$ pathology than $A \beta 1-42$ concentration $[19,20]$, into the calculation of the ES. This can perhaps explain, why some subjects categorized as neurochemically improbable AD 
based on the three available CSF biomarkers $(\mathrm{ES}=0$ or 1$)$, show progression in neuroimaging modalities (see fig. 1c and d). Certainly, some of these subjects would move to the neurochemically possible $\mathrm{AD}$ category $(\mathrm{ES}=2$ or 3$)$, if their $\mathrm{A} \beta 42 / 40$ ratio had been available and turned out pathologic. Nevertheless it must be stressed that even in the absence of A $\beta 42 / 40$ ratio, the interpretation based on the ES performed very well in this validation study.

The findings of this study outline a new property of the ES that has not been demonstrated before, namely its predictive features of disease progression rates in MCI. This adds to the value of the algorithm that has previously been shown, including: (a) precise estimation of risk to develop dementia in pre-dementia AD. (b) Allowing inter-laboratory comparisons of lab test results, despite use of different assays and biomarker cut-offs between centers. (c) Interpretation and presentation of all possible biomarker patterns, including those that are inconsistent with hallmark AD-like biomarker patterns.

\section{Acknowledgements}

Data collection and sharing for this project was funded by the Alzheimer's Disease Neuroimaging Initiative (ADNI) (National Institutes of Health Grant U01 AG024904) and DOD ADNI (Department of Defense award number W81XWH-12-2-0012). ADNI is funded by the National Institute on Aging, the National Institute of Biomedical Imaging and Bioengineering, and through generous contributions from the following: Alzheimer's Association; Alzheimer's Drug Discovery Foundation; Araclon Biotech; BioClinica, Inc.; Biogen Idec Inc.; Bristol-Myers Squibb Company; Eisai Inc.; Elan Pharmaceuticals, Inc.; Eli Lilly and Company; EuroImmun; F. Hoffmann-La Roche Ltd and its affiliated company Genentech, Inc.; Fujirebio; GE Healthcare; IXICO Ltd.; Janssen Alzheimer Immunotherapy 
Research \& Development, LLC.; Johnson \& Johnson Pharmaceutical Research \&

Development LLC.; Medpace, Inc.; Merck \& Co., Inc.; Meso Scale Diagnostics, LLC.;

NeuroRx Research; Neurotrack Technologies; Novartis Pharmaceuticals Corporation; Pfizer

Inc.; Piramal Imaging; Servier; Synarc Inc.; and Takeda Pharmaceutical Company. The

Canadian Institutes of Rev December 5, 2013 Health Research is providing funds to support

ADNI clinical sites in Canada. Private sector contributions are facilitated by the Foundation

for the National Institutes of Health (www.fnih.org). The grantee organization is the Northern

California Institute for Research and Education, and the study is coordinated by the

Alzheimer's Disease Cooperative Study at the University of California, San Diego. ADNI data

are disseminated by the Laboratory for Neuro Imaging at the University of Southern

California. The study was supported by grants from the Swedish and European Research

Councils, Swedish State Support for Clinical Research (ALFGBG), Frimurarstiftelsen,

Hjärnfonden, Alzheimerfonden, and the Torsten Söderberg Foundation. HZ is a Wallenberg

Academy Fellow. PL received research support from the Innovative Medicines Initiative Joint

Undertaking under EMIF grant agreement $\mathrm{n}^{\circ} 115372$, resources of which are composed of financial contribution from the European Union's Seventh Framework Programme (FP7/20072013) and EFPIA companies' in kind contribution;

\section{Competing interests:}

HZ has served at advisory boards of Roche Diagnostics, Eli Lilly and Wave, has received travel support from Teva and is a co-founder of Brain Biomarker Solutions in Gothenburg $\mathrm{AB}$, a GU Ventures-based platform company at the University of Gothenburg. KB has served as a consultant or at advisory boards for Alzheon, BioArctic, Biogen, Eli Lilly, Fujirebio Europe, IBL International, Merck, Novartis, Pfizer, and Roche Diagnostics, and is a cofounder of Brain Biomarker Solutions in Gothenburg AB, a GU Venture-based platform 
company at the University of Gothenburg. PL received consultation and lectures honoraria

from Innogenetics/Fujirebio Europe, IBL International, AJ Roboscreen, and Roche.

\section{References}

[1] Henriques AD, Benedet AL, Camargos EF, Rosa-Neto P, Nobrega OT (2018) Fluid and imaging biomarkers for Alzheimer's disease: Where we stand and where to head to. Exp Gerontol.

[2] Frisoni GB, Boccardi M, Barkhof F, Blennow K, Cappa S, Chiotis K, Demonet JF, Garibotto V, Giannakopoulos P, Gietl A, Hansson O, Herholz K, Jack CR, Jr., Nobili F, Nordberg A, Snyder HM, Ten Kate M, Varrone A, Albanese E, Becker S, Bossuyt P, Carrillo MC, Cerami C, Dubois B, Gallo V, Giacobini E, Gold G, Hurst S, Lonneborg A, Lovblad KO, Mattsson N, Molinuevo JL, Monsch AU, Mosimann U, Padovani A, Picco A, Porteri C, Ratib O, Saint-Aubert L, Scerri C, Scheltens P, Schott JM, Sonni I, Teipel S, Vineis P, Visser PJ, Yasui Y, Winblad B (2017) Strategic roadmap for an early diagnosis of Alzheimer's disease based on biomarkers. Lancet Neurol 16, 661-676.

[3] Lewczuk P, Riederer P, O'Bryant SE, Verbeek MM, Dubois B, Visser PJ, Jellinger KA, Engelborghs S, Ramirez A, Parnetti L, Jack CR, Jr., Teunissen CE, Hampel H, Lleo A, Jessen F, Glodzik L, de Leon MJ, Fagan AM, Molinuevo JL, Jansen WJ, Winblad B, Shaw LM, Andreasson U, Otto M, Mollenhauer B, Wiltfang J, Turner MR, Zerr I, Handels R, Thompson AG, Johansson G, Ermann N, Trojanowski JQ, Karaca I, Wagner H, OeckI P, van Waalwijk van Doorn L, Bjerke M, Kapogiannis D, Kuiperij HB, Farotti L, Li Y, Gordon BA, Epelbaum S, Vos SJB, Klijn CJM, Van Nostrand WE, Minguillon C, Schmitz M, Gallo C, Lopez Mato A, Thibaut F, Lista S, Alcolea D, Zetterberg H, Blennow K, Kornhuber J (2018) Cerebrospinal fluid and blood biomarkers for neurodegenerative dementias: An update of the Consensus of the Task Force on Biological Markers in Psychiatry of the World Federation of Societies of Biological Psychiatry. World J Biol Psychiatry 19, 244-328.

[4] Barnett JH, Lewis L, Blackwell AD, Taylor M (2014) Early intervention in Alzheimer's disease: a health economic study of the effects of diagnostic timing. BMC Neurol 14, 101.

[5] Blennow K, Hampel H, Weiner M, Zetterberg H (2010) Cerebrospinal fluid and plasma biomarkers in Alzheimer disease. Nat Rev Neurol 6, 131-144.

[6] Skillback T, Farahmand BY, Rosen C, Mattsson N, Nagga K, Kilander L, Religa D, Wimo A, Winblad B, Schott JM, Blennow K, Eriksdotter M, Zetterberg H (2015) Cerebrospinal fluid tau and amyloid-beta142 in patients with dementia. Brain 138, 2716-2731.

[7] Kuhlmann J, Andreasson U, Pannee J, Bjerke M, Portelius E, Leinenbach A, Bittner T, Korecka M, Jenkins RG, Vanderstichele H, Stoops E, Lewczuk P, Shaw LM, Zegers I, Schimmel H, Zetterberg H, Blennow K (2017) CSF Abeta1-42 - an excellent but complicated Alzheimer's biomarker - a route to standardisation. Clin Chim Acta 467, 27-33.

[8] Lewczuk P, Zimmermann R, Wiltfang J, Kornhuber J (2009) Neurochemical dementia diagnostics: a simple algorithm for interpretation of the CSF biomarkers. J Neural Transm (Vienna) 116, 1163-1167.

[9] Hansson O, Zetterberg H, Buchhave P, Londos E, Blennow K, Minthon L (2006) Association between CSF biomarkers and incipient Alzheimer's disease in patients with mild cognitive impairment: a followup study. Lancet Neurol 5, 228-234.

[10] Shaw LM, Vanderstichele H, Knapik-Czajka M, Figurski M, Coart E, Blennow K, Soares H, Simon AJ, Lewczuk P, Dean RA, Siemers E, Potter W, Lee VM, Trojanowski JQ (2011) Qualification of the analytical and clinical performance of CSF biomarker analyses in ADNI. Acta Neuropathol 121, 597-609.

[11] Lewczuk P, Kornhuber J, Toledo JB, Trojanowski JQ, Knapik-Czajka M, Peters O, Wiltfang J, Shaw LM (2015) Validation of the Erlangen Score Algorithm for the Prediction of the Development of Dementia due to Alzheimer's Disease in Pre-Dementia Subjects. J Alzheimers Dis 48, 433-441.

[12] Filipek-Gliszczynska A, Barczak A, Budziszewska M, Mandecka M, Gabryelewicz T, Barcikowska M (2018) The Erlangen Score Algorithm in the diagnosis and prediction of the progression from subjective cognitive decline and mild cognitive impairment to Alzheimer-type dementia. Folia Neuropathol 56, 88-96.

[13] Baldeiras I, Santana I, Leitao MJ, Vieira D, Duro D, Mroczko B, Kornhuber J, Lewczuk P (2019) Erlangen Score as a tool to predict progression from mild cognitive impairment to dementia in Alzheimer's disease. Alzheimers Res Ther 11, 2. 
[14] Shaw LM, Vanderstichele H, Knapik-Czajka M, Clark CM, Aisen PS, Petersen RC, Blennow K, Soares H, Simon A, Lewczuk P, Dean R, Siemers E, Potter W, Lee VM, Trojanowski JQ (2009) Cerebrospinal fluid biomarker signature in Alzheimer's disease neuroimaging initiative subjects. Ann Neurol 65, 403-413.

[15] Jack CR, Jr., Bernstein MA, Fox NC, Thompson P, Alexander G, Harvey D, Borowski B, Britson PJ, J LW, Ward C, Dale AM, Felmlee JP, Gunter JL, Hill DL, Killiany R, Schuff N, Fox-Bosetti S, Lin C, Studholme C, DeCarli CS, Krueger G, Ward HA, Metzger GJ, Scott KT, Mallozzi R, Blezek D, Levy J, Debbins JP, Fleisher AS, Albert M, Green R, Bartzokis G, Glover G, Mugler J, Weiner MW (2008) The Alzheimer's Disease Neuroimaging Initiative (ADNI): MRI methods. J Magn Reson Imaging 27, 685-691.

[16] Fischl B, Salat DH, Busa E, Albert M, Dieterich M, Haselgrove C, van der Kouwe A, Killiany R, Kennedy D, Klaveness S, Montillo A, Makris N, Rosen B, Dale AM (2002) Whole brain segmentation: automated labeling of neuroanatomical structures in the human brain. Neuron 33, 341-355.

[17] Fischl B, van der Kouwe A, Destrieux C, Halgren E, Segonne F, Salat DH, Busa E, Seidman L, Goldstein J, Kennedy D, Caviness V, Makris N, Rosen B, Dale AM (2004) Automatically parcellating the human cerebral cortex. Cereb Cortex 14, 11-22.

[18] Jack CR, Jr., Knopman DS, Jagust WJ, Petersen RC, Weiner MW, Aisen PS, Shaw LM, Vemuri P, Wiste HJ, Weigand SD, Lesnick TG, Pankratz VS, Donohue MC, Trojanowski JQ (2013) Tracking pathophysiological processes in Alzheimer's disease: an updated hypothetical model of dynamic biomarkers. Lancet Neurol 12, 207-216.

[19] Lewczuk P, Matzen A, Blennow K, Parnetti L, Molinuevo JL, Eusebi P, Kornhuber J, Morris JC, Fagan AM (2017) Cerebrospinal Fluid Abeta42/40 Corresponds Better than Abeta42 to Amyloid PET in Alzheimer's Disease. J Alzheimers Dis 55, 813-822.

[20] Lewczuk P, Lelental N, Spitzer P, Maler JM, Kornhuber J (2015) Amyloid-beta 42/40 cerebrospinal fluid concentration ratio in the diagnostics of Alzheimer's disease: validation of two novel assays. $J$ Alzheimers Dis 43, 183-191. 
Table 1 Demographics and the cognitive and neuroimaging results. M/F, number of the male/female subjects; Mdn., median; IQR, inter-quartile range; Std. dev., standard deviation; MMSE, mini-mental state examination; ADAS-Cog, Alzheimer's Disease Assessment Scale-cognitive subscale; E3, *10 $30^{3} \mathrm{E} 6, * 10^{6}$. All measures were collected at the baseline.

\begin{tabular}{|c|c|c|c|c|c|c|c|c|c|c|c|}
\hline \multirow[t]{2}{*}{ Erlangen score } & \multirow{2}{*}{$\begin{array}{l}\text { Sex } \\
M / F\end{array}$} & \multirow{2}{*}{$\begin{array}{l}\text { Age (years) } \\
\text { Mean (Std.dev.) }\end{array}$} & \multirow[b]{2}{*}{ Mdn (IQR) } & \multirow{2}{*}{$\begin{array}{l}\text { MMSE (points) } \\
\text { Mean (Std.dev.) }\end{array}$} & \multirow[b]{2}{*}{$\begin{array}{l}\text { Mdn } \\
\text { (IQR) }\end{array}$} & \multicolumn{2}{|c|}{ ADAS-Cog (points) } & \multicolumn{2}{|c|}{ Whole brain volume (mm3) } & \multicolumn{2}{|c|}{ Hippocampal volumes (mm3) } \\
\hline & & & & & & Mean (Std.dev.) & Mdn (IQR) & Mean (Std.dev.) & Mdn (IQR) & Mean (Std.dev.) & Mdn (IQR) \\
\hline $0-1$ & $12 / 35$ & $73.8(7.9)$ & $74(67.5-80.4)$ & $27.4(1.7)$ & $28(26-29)$ & $14.8(5.4)$ & $15.8(11.3-17.3)$ & $1.05 E 6(.12 E 6)$ & $1.05 E 6(0.98 E 6-1.08 E 6)$ & $6.98 E 3(1.28 E 3)$ & $7.20 E 3(6.05 E 3-7.68 E 3)$ \\
\hline $2-3$ & $6 / 28$ & $76.8(7.5)$ & $77.2(73.9-80.9)$ & $26.4(1.9)$ & $26(25-28)$ & $20.3(6.2)$ & $19.9(15.7-24.7)$ & $1.04 E 6(0.12 E 6)$ & $1.07 E 6(0.96 E 6-1.13 E 6)$ & $6.26 E 3(1.28 E 3)$ & $6.50 E 3(5.34 E 3-7.11 E 3)$ \\
\hline 4 & $45 / 67$ & $78.1(7.1)$ & $79.1(70.3-85.9)$ & $26.9(1.8)$ & $27(25-28)$ & $20.3(6.0)$ & $21(16.3-24.3)$ & $0.99 E 6$ (0.10E6) & $0.98 E 6$ (0.92E6-1.05E6) & $6.20 E 3(0.87 E 3)$ & $6.11 E 3(5.57 E 3-6.75 E 3)$ \\
\hline
\end{tabular}


Table 2 Participants at follow-up times

\begin{tabular}{lccccccccccc} 
Follow-up & & Baseline & $\begin{array}{c}\mathbf{6} \\
\text { months }\end{array}$ & $\begin{array}{c}\mathbf{1 2} \\
\text { months }\end{array}$ & $\begin{array}{c}\mathbf{1 8} \\
\text { months }\end{array}$ & $\begin{array}{c}\mathbf{2 4} \\
\text { months }\end{array}$ & $\begin{array}{c}\mathbf{3 0} \\
\text { months }\end{array}$ & $\begin{array}{c}\mathbf{3 6} \\
\text { months }\end{array}$ & $\begin{array}{c}\mathbf{4 2} \\
\text { months }\end{array}$ & $\begin{array}{c}\mathbf{4 8} \\
\text { months }\end{array}$ & $\begin{array}{c}\mathbf{5 4} \\
\text { months }\end{array}$ \\
\hline $\begin{array}{l}\text { Erlangen } \\
\text { score }\end{array}$ & $\mathbf{0}$ or $\mathbf{1}$ & 47 & 45 & 43 & 40 & 36 & 33 & 30 & 21 & 6 & 3 \\
& $\mathbf{2}$ or $\mathbf{3}$ & 34 & 33 & 31 & 29 & 27 & 27 & 22 & 10 & 5 & 3 \\
& $\mathbf{4}$ & 112 & 108 & 106 & 98 & 91 & 81 & 75 & 36 & 16 & 10 \\
\hline
\end{tabular}




\section{Figure legends}

Fig. 1. Correlations between follow up time and the neuropsychologic and neuroimaging population-averaged (marginal) metrics in the three ES categories:

1a. MMSE score change from the baseline;

1b. ADAS-Cog score change from the baseline;

1c. whole brain volume change from the baseline;

1d. hippocampal volume change from the baseline.

Figure 1.
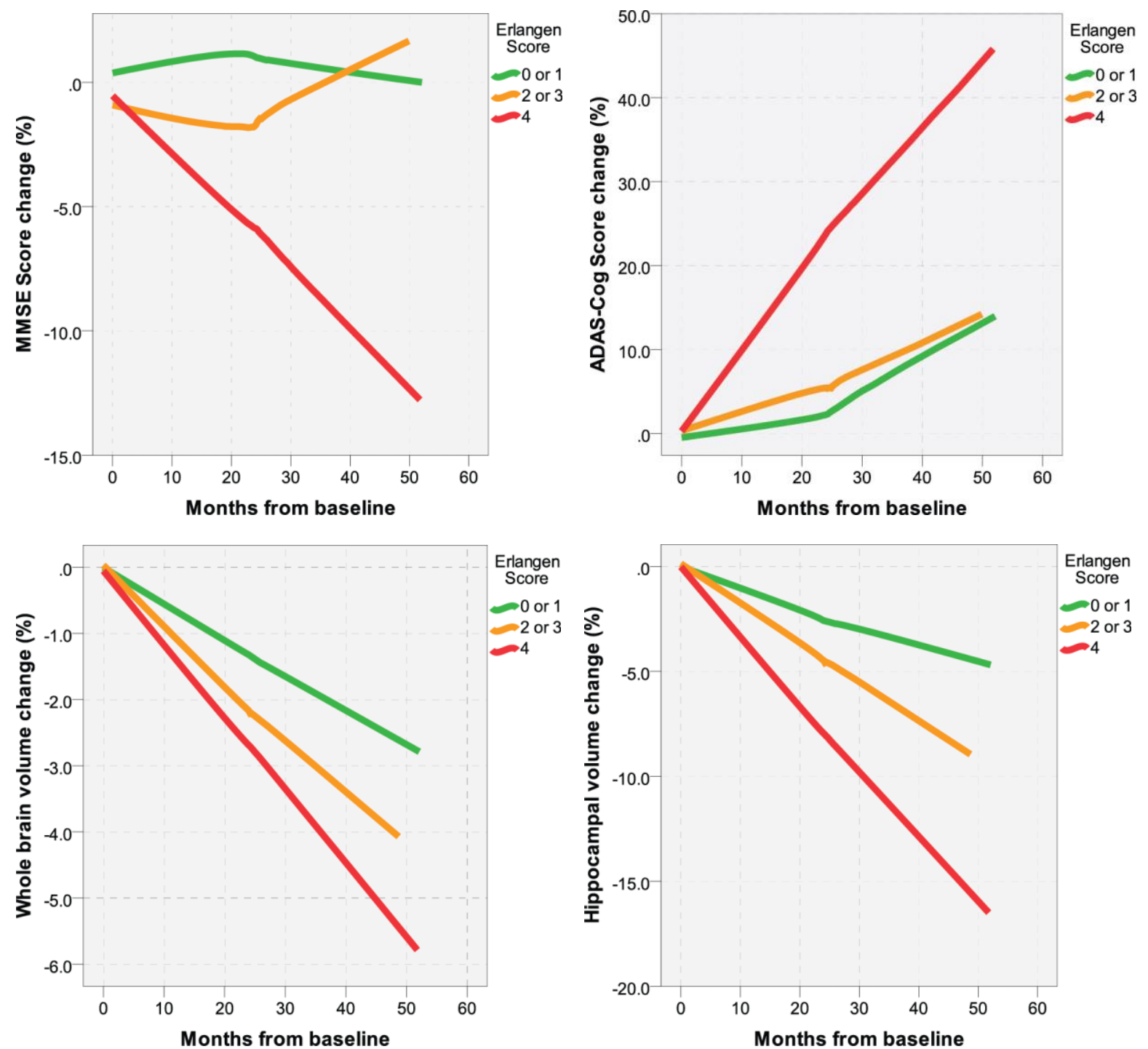